\title{
CUIDADO FAMILIAR NA ADESÃO À TERAPIA ANTIRRETROVIRAL EM CRIANÇAS COM HIV/AIDS
}

Tassiana Potrich ${ }^{1}$, Cristiane Cardoso de Paula², Stela Maris de Mello Padoin ${ }^{3}$, Clarissa Bohrer da Silva ${ }^{4}$

RESUMO: Objetivou-se avaliar as evidências disponíveis nos artigos científicos sobre como o cuidado familiar interfere na adesão à terapia antirretroviral em crianças com HIV/aids. Trata-se de revisão integrativa desenvolvida em abril de 2012 nas bases de dados Lilacs e Medline utilizando-se os descritores [HIV or aids] and [criança] and [adesão a medicação or terapeutica or terapia anti-retroviral de alta atividade or anti-retrovirais]. Totalizaram 8 artigos analisados. Evidenciou-se que o cuidado familiar interfere positivamente quando: têm acesso gratuito aos antirretrovirais; não revelaram o diagnóstico; contam com apoio; conferem a ingestão; usam do medo de morrer; utilizam estratégias para facilitar a ingestão. São situações que interferem negativamente no cuidado: estresse; culpabilização; cuidado institucional; negligência; dificuldade em compreender o tratamento; orfandade; situações socioeconômicas precárias. A atuação da equipe de saúde, por meio de orientação coerente com o cotidiano e com o nível de compreensão familiar, é imprescindível na tentativa de intervir eficazmente na adesão.

PALAVRAS-CHAVE: Síndrome de imunodeficiência adquirida; Criança; Família; Adesão à medicação; Terapia antirretroviral de alta atividade.

\section{FAMILY CARE IN COMPLIANCE WITH ANTIRETROVIRAL THERAPY IN CHILDREN WITH HIVIAIDS}

ABSTRACT: The aim was to evaluate the evidence available in scientific articles on how family care influences compliance with anti-retroviral therapy in children with HIV/aids. It is an integrative review undertaken in April 2012 using the databases Lilacs and Medline using the descriptors [HIV or AIDS] and [child] and [compliance with medication or therapy or high activity antiretroviral therapy or antiretrovirals]. A total of 8 articles was analyzed. It was evidenced that family care has a positive influence when: there is free access to antiretrovirals; the diagnosis was not revealed; the family could rely on support; they check the ingestion; use the fear of dying; they use strategies for facilitating ingestion. The following situations influence the care negatively: stress; blaming; institutional care; neglect; difficulty in understanding the treatment; being an orphan; weak socio-economic situations. The work of the health team, through guidance which is consistent with the routine and the level of family understanding, is essential in the attempt to interfere efficaciously in the issue of adherence.

KEY-WORDS: Acquired Immunodeficiency Syndrome; Child; Family; Adherence to medication; High activity antiretroviral therapy.

\section{CUIDADO FAMILIAR EN LA ADHESIÓN A LA TERAPIA ANTIRRETROVIRAL EN NIÑOS CON HIV/SIDA}

RESUMEN: El objetivo fueevaluarlas evidencias disponibles en los artículos científicos sobre cómoel cuidado familiarinterfiereenlaadhesión a la terapiaantirretroviral enniños conHIV/SIDA. Es una revisión integrativa desarrollada en abril de 2012 en las bases de datos Lilacs y Medline, utilizándose los descriptores [HIV or aids] and [criança] and [adesão a medicação or terapeutica or terapia anti-retroviral dealta atividade or antiretrovirais]. Fueronanalizados el total deochoartículos. Seevidenció que el cuidado familiarinterfiere de forma positiva cuando: hay acceso gratuito a los antirretrovirais; no se revela el diagnóstico; hay apoyo; hacen la conferencia de la ingestión; usan del miedo de morir; utilizan estrategias para facilitarlaingestión. Sonsituacionesqueinterfierennegativamenteenel cuidado: estrese; atribuirculpa; cuidadoinstitucional; negligencia; dificultad decomprender el tratamiento; huerfanidad; situaciones socioeconómicas precarias. La actuación del equipo de salud, pormedio de orientación coherente con el cotidiano y con el nivel de comprensión familiar, es imprescindible en la tentativa de intervenir de modo eficaz en la adhesión. DESCRIPTORES: Síndrome de inmunodeficiencia adquirida; Niño; Familia; Adhesión a medicación; Terapia antirretroviral de alta actividad.

\footnotetext{
${ }^{1}$ Enfermeira. Mestranda pelo Programa de Pós-Graduação em Enfermagem da Universidade Federal de Santa Maria. Santa Maria UFSM. Membro do Grupo de Pesquisa Cuidado à Saúde das Pessoas, Famílias e Sociedade.

${ }^{2}$ Enfermeira. Doutora em Enfermagem. Professora do Departamento e do Programa de Pós-Graduação em de Enfermagem da UFSM. Vice-Líder do Grupo de Pesquisa Cuidado à Saúde das Pessoas, Famílias e Sociedade.

${ }^{3}$ Enfermeira. Doutora em Enfermagem. Professora do Departamento e do Programa de Pós-Graduação em de Enfermagem da UFSM. Líder do Grupo de Pesquisa Cuidado à Saúde das Pessoas, Famílias e Sociedade.

${ }^{4}$ Enfermeira. Membro do Grupo de Pesquisa Cuidado à Saúde das Pessoas, Famílias e Sociedade.
}

Autor correspondente:

Recebido: 14/09/2012

Cristiane Cardoso de Paula

Aprovado: 30/11/2012

Universidade Federal de Santa Maria

Av. Roraima, 1000 - 97105-900 - Santa Maria-RS-brasil

E-mail: cris_depaula1@hotmail.com 


\section{INTRODUÇÃO}

A Síndrome da Imunodeficiência Adquirida (aids), doença causada pela infecção do Vírus da Imunodeficiência Humana (HIV), configura-se como um dos maiores problemas de saúde pública no mundo ${ }^{(1)}$. Tal fato se justifica por esta ser uma doença crônica, sem cura, pela vulnerabilidade para se proteger da infecção pelo HIV e do adoecimento pela aids, somados ao desafio da adesão à Terapia Antirretroviral (TARV), fator determinante para a redução da morbidade e mortalidade.

Atualmente, a aids apresenta um perfil epidemiológico modificado, destacando-se o processo de feminização da epidemia ${ }^{(2)}$. Uma das consequências desse processo é o aumento de casos de crianças infectadas pela categoria de exposição à transmissão vertical do vírus. Em 2011, houve no Brasil 148 notificações de crianças expostas ao HIV por transmissão vertical e 104 casos de notificação de crianças com diagnóstico confirmado de aids por essa categoria, representando 90,4\% das notificações em menores de 13 anos de idade ${ }^{(3)}$. Dessa forma, a criança com HIV/aids ganha importância no contexto da epidemia, sendo que se vivencia a primeira geração de crianças e adolescentes infectadas desde o nascimento ${ }^{(4)}$.

Essas vivenciam um processo de cronicidade da doença, tendo seu cotidiano modificado pelo tratamento que contempla o acompanhamento permanente em serviços de saúde, uso contínuo de medicamentos, limitações físicas devido aos sinais e sintomas e a necessidade de reinternações hospitalares ${ }^{(5)}$. Além disso, vivenciam um contexto que interfere na estrutura econômica e psicoemocional familiar, no qual muitas vezes enfrentam a orfandade do(s) pai(s) e dificuldades socioeconômicas ${ }^{(6)}$. A criança depende de seus familiares, especialmente no que se refere ao cuidado medicamentoso, sendo os responsáveis por administrar as medicações conforme a prescrição e garantir a adesão $0^{(7)}$.

Dessa forma, a enfermagem necessita desenvolver ações de intervenção de modo que possa viabilizar acompanhamento integral de seu crescimento e desenvolvimento e da condição sorológica e suas especificidades, contemplando a criança e sua família. Nessa perspectiva, tem-se como objetivo avaliar as evidências disponíveis nos artigos científicos sobre como o cuidado fa- miliar interfere na adesão à TARV em crianças com HIV/aids.

\section{MÉTODO}

Foi realizada revisão integrativa da literatura percorrendo as seguintes etapas: identificação do tema, seleção da questão de pesquisa e objetivo da revisão, estabelecimento de critérios de inclusão e exclusão, seleção dos artigos a serem analisados, avaliação das evidências, análise e apresentação da síntese do conhecimento evidenciado ${ }^{(8)}$.

Estabeleceu-se a questão de pesquisa: como o cuidado da família interfere na adesão ao tratamento com TARV em crianças que têm HIV/ aids? A busca bibliográfica ocorreu em abril de 2012 e foi desenvolvida na Biblioteca Virtual de Saúde, na base de dados eletrônica Literatura Latino-Americana e do Caribe em Ciências da Saúde (LILACS) e na Medical Literature Analysis and Retrieval System Online (MEDLINE). Utilizaram-se os descritores "hiv" or "aids" and "crianca" and "adesão a medicacao" or "terapeutica" or "terapia anti-retroviral de alta atividade" or "anti-retrovirais".

Para selecioná-los os critérios de inclusão foram: artigos de pesquisa; disponíveis na íntegra online e gratuitamente; nos idiomas português, inglês ou espanhol. E como critérios de exclusão: artigos incompletos. Apuraram-se 243 produções e totalizaram 8 artigos na íntegra (Figura 1).

Após a leitura exaustiva dos estudos selecionados, foi preenchido um instrumento validado ${ }^{(9)}$ (Quadro 1). A análise das evidências foi classificada em sete níveis ${ }^{(10)}$.

\section{RESULTADOS}

Quanto à caracterização dos artigos analisados, no que se refere à procedência, verificou-se o Brasil com 62\% $(n=5)$. Em relação à área do conhecimento, constatou-se uma concentração da Medicina com 50\% ( $\mathrm{n}=4)$. Quanto ao ano de publicação evidenciou o ano de 2008, com 37,5\% $(n=3)$ e em relação ao delineamento predominaram, igualmente, 37,5\% ( $n=3)$ estudos com delineamento quantitativo não experimental e delineamento qualitativo. No que se refere à força das evidências predominaram estudos com nível de evidência $6(\mathrm{n}=6)$ (Tabela 1). 


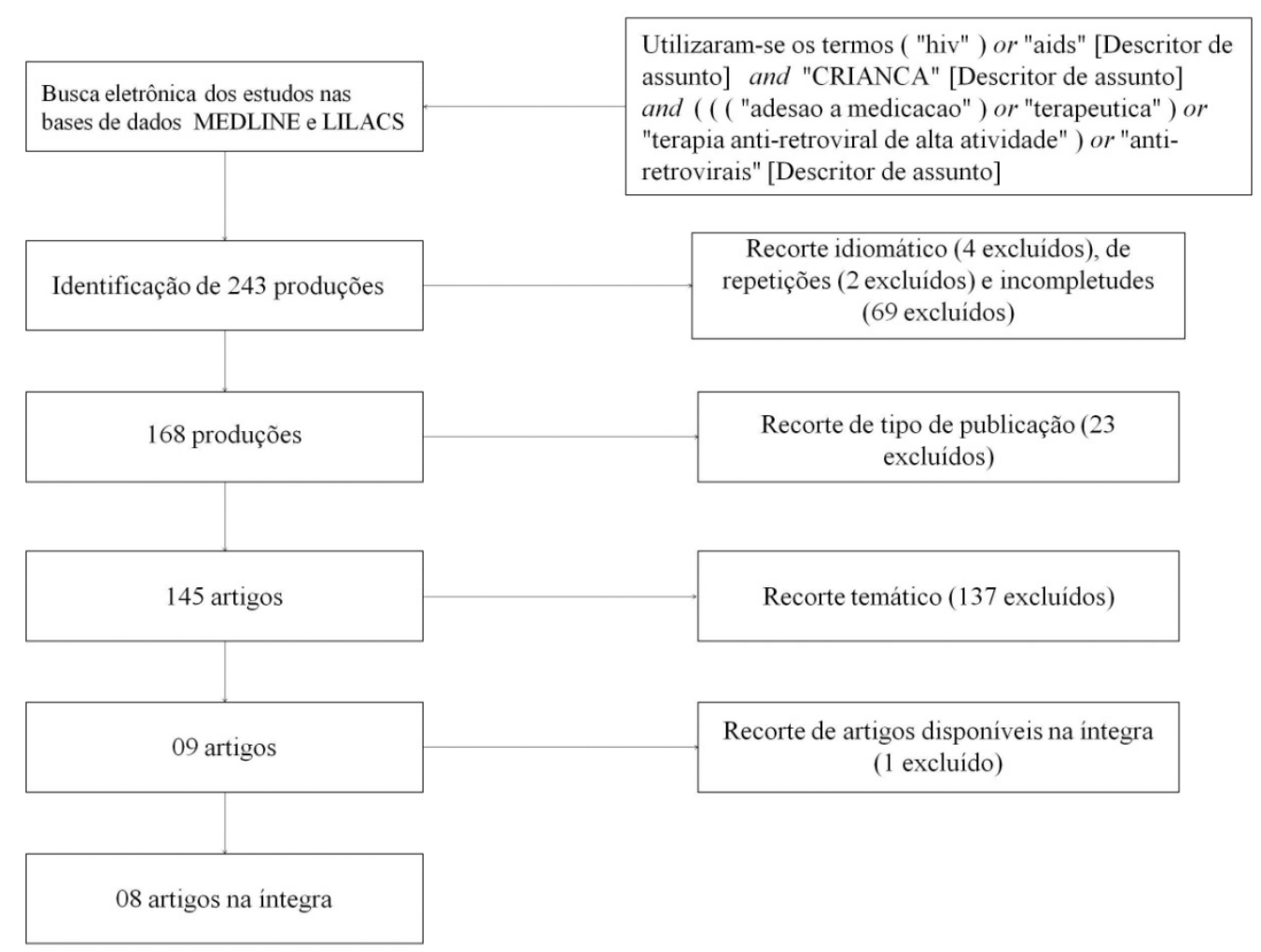

Figura 1 - Estrutura da seleção de estudos na temática cuidado familiar na adesão ao tratamento com terapia antirretroviral em crianças que têm HIV/aids, de acordo com os critérios de exclusão, nas bases de dados LILACS e MEDLINE. Santa Maria, 2012

Tabela 1 - Caracterização dos artigos analisados. LILACS, MEDLINE. Santa Maria, 2012

\begin{tabular}{lcc}
\hline Procedência & $\mathbf{n}$ & $\mathbf{\%}$ \\
Brasil & 5 & 62 \\
Jamaica & 1 & 12,5 \\
Etiópia & 1 & 12,5 \\
Dinamarca & 1 & 12,5 \\
Área do conhecimento & $\mathbf{n}$ & $\mathbf{\%}$ \\
Medicina & 4 & 50 \\
Enfermagem & 3 & 37,5 \\
Psicologia & 1 & 12,5 \\
Ano de publicação & $\mathbf{n}$ & $\mathbf{\%}$ \\
2007 & 1 & 12,5 \\
2008 & 3 & 37,5 \\
2009 & 2 & 25 \\
2010 & 1 & 12,5 \\
2011 & 1 & 12,5 \\
Delineamento da pesquisa & $\mathbf{n}$ & $\mathbf{\%}$ \\
Quantitativo não experimental & 3 & 37,5 \\
Qualitativo & 3 & 37,5 \\
Estudo de Coorte & 2 & 25 \\
Nível de evidência & $\mathbf{n}$ & $\mathbf{\%}$ \\
4 & 2 & 25 \\
6 & 6 & 75 \\
Total & $\mathbf{8}$ & $\mathbf{1 0 0}$ \\
\hline
\end{tabular}


Quadro 1 - Resultados de estudos na temática do cuidado familiar na adesão do tratamento com terapia antirretroviral em crianças que tem HIV/aids, nas bases de dados LILACS e MEDLINE. Santa Maria, 2012

\begin{tabular}{|c|c|c|c|c|}
\hline Referência & $\begin{array}{c}\text { Área/ } \\
\text { Procedência }\end{array}$ & Objetivo & $\begin{array}{c}\text { Delineamento/ } \\
\text { Nível de Evidência }\end{array}$ & Principais Resultados \\
\hline (16) & $\begin{array}{l}\text { Medicina/ } \\
\text { Jamaica }\end{array}$ & $\begin{array}{l}\text { Descrever os pa- } \\
\text { drões de adesão à } \\
\text { TARV. }\end{array}$ & Coorte/4 & $\begin{array}{l}\text { Crianças em cuidados residenciais têm } \\
\text { maior adesão em comparação com as } \\
\text { em cuidado familiar. A não aderência foi } \\
\text { relacionada principalmente ao estresse. }\end{array}$ \\
\hline (10) & $\begin{array}{l}\text { Medicina/ } \\
\text { Etiopia }\end{array}$ & $\begin{array}{l}\text { Determinar as } \\
\text { taxas e os fatores } \\
\text { associados à ade- } \\
\text { são à TARV. }\end{array}$ & Quantitativo/6 & $\begin{array}{l}\text { Contribuiram para maior adesão: quando } \\
\text { a medicação não era paga pelos pais; a } \\
\text { não divulgação da doença para a criança; } \\
\text { não saber do problema de saúde do seu } \\
\text { cuidador. }\end{array}$ \\
\hline (17) & $\begin{array}{l}\text { Medicina/ } \\
\text { Dinamarca }\end{array}$ & $\begin{array}{l}\text { Descrever a eficá- } \\
\text { cia da TARV na } \\
\text { população. }\end{array}$ & Coorte/4 & $\begin{array}{l}\text { Em crianças menores a negligência dos } \\
\text { cuidadores é considerada motivo para a } \\
\text { baixa adesão. }\end{array}$ \\
\hline (12) & $\begin{array}{l}\text { Enfermagem/ } \\
\text { Brasil }\end{array}$ & $\begin{array}{l}\text { Conhecer o modo } \\
\text { de viver e as im- } \\
\text { plicações do uso } \\
\text { da TARV. }\end{array}$ & Qualitativo/6 & $\begin{array}{l}\text { Trabalho multidisciplinar com profissio- } \\
\text { nais da saúde e da educação. Auxílio de } \\
\text { familiares. Cuidador permitir autonomia } \\
\text { da criança na ingestão da medicação, po- } \\
\text { rém apoiando e conferindo. A mãe utiliza } \\
\text { o medo da morte para facilitar a ingestão } \\
\text { de medicação. }\end{array}$ \\
\hline (18) & $\begin{array}{l}\text { Medicina/ } \\
\text { Brasil }\end{array}$ & $\begin{array}{l}\text { Descrever as ma- } \\
\text { nifestações clíni- } \\
\text { cas da infecção. }\end{array}$ & Quantitativo/6 & $\begin{array}{l}\text { Responsáveis não souberam identificar a } \\
\text { TARV. A maioria eram cuidadores o que } \\
\text { demonstra o distanciamento de um dos } \\
\text { pais ou de ambos, ou reflete a perda por } \\
\text { óbito ou incapacidade de cuidarem dos } \\
\text { filhos ou ainda pela rejeição. }\end{array}$ \\
\hline (15) & $\begin{array}{l}\text { Psicologia/ } \\
\text { Brasil }\end{array}$ & $\begin{array}{l}\text { Identificar a pre- } \\
\text { valência de ade- } \\
\text { são à TARV e fa- } \\
\text { tores associados à } \\
\text { não adesão. }\end{array}$ & Quantitativo/6 & $\begin{array}{l}\text { A não adesão está relacionada a pouca } \\
\text { escolaridade e renda abaixo de um salá- } \\
\text { rio mínimo, idade avançada do cuidador } \\
\text { (avó), bem como à desintegração do meio } \\
\text { familiar (mãe solteira ou viúva, alto grau } \\
\text { de pobreza). }\end{array}$ \\
\hline (13) & $\begin{array}{l}\text { Enfermagem/ } \\
\text { Brasil }\end{array}$ & $\begin{array}{l}\text { Descrever e ana- } \\
\text { lisar a dimensão } \\
\text { matemática do } \\
\text { cuidado medica- } \\
\text { mentoso. }\end{array}$ & Qualitativo/6 & $\begin{array}{l}\text { A baixa escolaridade e o processo de } \\
\text { pauperização contribuem para o cuidado } \\
\text { irregular. Retiram os rótulos dos frascos } \\
\text { gerando risco de troca e de erros rela- } \\
\text { cionados à administração. Substituem o } \\
\text { nome de um antirretroviral por vitamina } \\
\text { para manter a criança fora desse diálogo. }\end{array}$ \\
\hline (14) & $\begin{array}{l}\text { Enfermagem/ } \\
\text { Brasil }\end{array}$ & $\begin{array}{l}\text { Conhecer os fa- } \\
\text { tores que inter- } \\
\text { ferem na adesão } \\
\text { à TARV a partir } \\
\text { do relato de cui- } \\
\text { dadores. }\end{array}$ & Qualitativo/6 & $\begin{array}{l}\text { Predomina entre os cuidadores: a mãe; } \\
\text { baixo nível de escolaridade e socioeco- } \\
\text { nômico. Dificuldade na compreensão } \\
\text { das metas da terapia. A adesão pode ser } \\
\text { comprometida pelo esquecimento ou a } \\
\text { administração fora do domicílio. O ma- } \\
\text { nejo dos efeitos colaterais por meio de } \\
\text { estratégias para facilitar a administração. }\end{array}$ \\
\hline
\end{tabular}




\section{DISCUSSÃO}

\section{Quando o cuidado familiar interfere positivamente na adesão à TARV}

Ao analisar a produção científica foi possível evidenciar alguns aspectos que interferem positivamente na adesão à TARV. O acesso gratuito aos ARVs facilita a adesão da criança com HIV/aids, uma vez que os cuidadores têm renda proveniente de benefícios recebidos do governo ${ }^{(19)}$. Essas crianças, em sua maioria, pertencem a grupos desfavorecidos no aspecto econômico e seus cuidadores apresentam baixo nível de escolaridade ${ }^{(19-20)}$.

Esse fator, somado à ausência de trabalho remunerado, configura-se como um dificultador no acesso aos ARVs. Destaca-se a política pública brasileira de enfrentamento da epidemia de aids, que é referência mundial pelo acesso gratuito ao tratamento ${ }^{(21)}$.

A não divulgação da condição sorológica da criança, assim como crianças que não estavam cientes do problema de saúde de seu familiar cuidador, compuseram aspectos que facilitaram a adesão à TARV, achado que é convergente a estudo internacional ${ }^{(11)}$. Porém, estudos nacionais apontam que o desconhecimento da situação sorológica pela criança dificulta a compreensão da doença e limita o poder de transformação de seus comportamentos e atitudes, impactando na adesão à medicação ${ }^{(6)}$.

Quanto ao apoio dos profissionais de saúde e da educação, compartilhar informações, vivências e experiências, contribui para o desenvolvimento do empoderamento, amenizando o processo estressante que a situação acarreta aos cuidadores ${ }^{(22)}$.

A importância dos familiares apoiarem e conferirem a ingestão dos medicamentos sem dar completa autonomia à criança converge com a fase do desenvolvimento, em que ainda não possuem autonomia suficiente para gerenciar o cuidado de si. Assim, o cuidador é o responsável por colocar o comportamento da criança sob controle das contingências relativas à terapia medicamentosa ${ }^{(23)}$.

Ao deparar-se com a possibilidade de não adesão, muitas vezes o familiar se utiliza do medo que a criança tem da morte, uma vez que, o mínimo de informações é suficiente para compreender que sem a realização do tratamento sua chance de vida é pequena. Nestes casos, aderem ao tratamento proposto por obrigação ou medo da morte ${ }^{(24)}$.

Outro fato evidenciado foi o esquecimento de ad- ministrar as doses nos horários corretos e a ingestão de medicação fora do ambiente familiar. Ambos instigaram estratégias para facilitar a administração de medicamentos e minimizar os efeitos colaterais. Diante de dificuldades da rotina do tratamento os familiares se utilizam de estratégias para que a criança tome o medicamento, garantindo a continuidade do tratamento ${ }^{(25)}$.

\section{Quando o cuidado familiar interfere negativamente na adesão à TARV}

Um fato evidenciado que interfere negativamente na adesão à TARV é quando o familiar é acometido pelo estresse devido à cronicidade da doença, uma vez que demanda tratamento permanente e a premência da revelação do diagnóstico ${ }^{(26)}$. Dessa forma, os familiares defrontam-se, diariamente, com uma rotina de vida repleta de estressores: ansiedade, problemas conjugais e financeiros, culpa e frustração, podendo trazer como consequência sobrecarga física e emocional ${ }^{(23)}$.

O processo de culpabilização na família ocorre tanto pela própria infecção quanto pelas dificuldades na adesão, responsabilizando-os pelo adoecimento da criança. O diagnóstico de aids somado a TARV, altera de forma significativa a vida da criança e a dinâmica familiar que, por sua vez, é reorganizada de acordo com o novo cotidiano ${ }^{(25)}$.

Outra evidência deu-se entre crianças em cuidado em casa de apoio institucional, no qual se garantiu a adesão, se comparadas àquelas sob cuidado familiar. Quando as crianças contam, exclusivamente, com o cuidado familiar pode haver repercussões negativas na adesão, uma vez que há convívio com muitas pessoas, em detrimento de um responsável pela administração dos medicamentos. Isso se potencializa em condição de pobreza, o que pode acarretar em contextos adversos que dificultam o uso dos medicamentos ${ }^{(23)}$.

Diante dessas situações de vulnerabilidade, muitas crianças com aids são encaminhadas para instituições de apoio, devido às condições socioeconômicas precárias, ou em consequência da desestruturação familiar ${ }^{(27)}$. Nesse sentido, as instituições cumprem um papel de apoio à saúde das crianças de acordo com sua capacidade. Porém, é necessário investir na qualificação da família, dando subsídios para que esta possa exercer o cuidado à criança como previsto legalmente. Ou seja, na convivência familiar e com a corresponsabilização do Estado, sociedade e família no cuidado ${ }^{(28)}$.

No que se refere à negligência em cumprir a 
prescrição, esta pode estar relacionada ao desconhecimento do familiar acerca do regime terapêutico e sua disponibilidade em mantê-lo ${ }^{(29)}$. A negligência ocorre por meio de doses incorretas ou omitidas, falta de precisão no tempo ou deficiência de medicamentos, representando um entrave para o sucesso terapêutico, e expõe a criança a riscos de saúde ${ }^{(30)}$.

Outro fato trata da identificação dos ARVs, em que os familiares apontam dificuldade de memorizar os nomes. Isso pode acontecer devido aos antirretrovirais apresentarem nomes complexos e extensos, de difícil pronúncia e assimilação. Sendo assim, precisam de apoio para adesão ${ }^{(31)}$, possibilitando a identificação correta dos ARVs e a compreensão quanto à ingestão, de forma a minimizar a confusão entre os medicamentos ${ }^{(32)}$.

Os familiares também se utilizam de estratégias de ocultamento da medicação, uma vez que ainda não revelaram o diagnóstico para a criança, de modo a garantir que ela não descubra por meio dos medicamentos. Soma-se a intenção de que outras pessoas também não descubram, no intuito de proteger a criança de situações de preconceito e de discriminação. Assim, o ocultamento do cotidiano medicamentoso pode estar presente na relação com a criança, nas relações internas da família e na interação com o meio social ${ }^{(33)}$. O conhecimento limitado da criança acerca de sua condição sorológica, é mantido e justificado pela imaturidade cognitiva e emocional da criança ${ }^{(34)}$.

Diante da orfandade ou rejeição pela família, o seu cotidiano de cuidado é modificado, podendo influenciar negativamente na adesão(35). Os familiares, normalmente, são os responsáveis pela estrutura emocional da criança, desempenhando o papel de facilitadores no seu processo de crescimento e desenvolvimento ${ }^{(27)}$. Também há situações de incapacidade de cuidar da criança, devido às dificuldades do próprio familiar em assumir essa responsabilidade. Isso interfere negativamente na adesão, pois poderão ocorrer esquecimentos, mudanças no cronograma do familiar e até a falta de medicamentos $^{(29)}$.

As instituições como espaços de cuidado começam a ser procuradas na medida em que vai sendo construída uma imagem de incapacidade da família em exercer esse cuidado. Essa situação de vulnerabilidade potencializa-se em condições de precariedade socioeconômica, por vezes, sendo rotuladas como desestruturadas de acordo com o discurso vigente na sociedade sobre a representação de família( ${ }^{(28)}$.

Em relação à baixa escolaridade, o grau de instrução limitado de familiares configura um fator que dificulta o processo de educação em saúde no cuidado ${ }^{(31)}$. Destaca-se que tal condição impõe dificuldades de acesso ao tratamento. Ainda, a idade avançada do cuidador interfere, especialmente. em relação ao processo de envelhecimento, quando os avós estão responsáveis pelo cuidado de crianças órfãs ${ }^{(25)}$.

A dificuldade do familiar de compreender o porquê e como fazer o tratamento repercute na adesão, sugerindo que estes não estejam sendo informados e esclarecidos ou que não estejam compreendendo e se apropriando das informações recebidas. Inclusive, as dificuldades de adesão podem sofrer influência da comunicação familiar precária sobre o diagnóstico e o tratamento ${ }^{(33)}$.

\section{CONCLUSÃO}

A produção científica evidenciou que o cuidado familiar pode influenciar tanto positivamente quanto sofrer a influência de fatores negativos para a adesão à TARV. A distribuição gratuita dos medicamentos. mantida pela política pública brasileira de enfrentamento da epidemia de aids é um dos facilitadores. A baixa renda, aliada à baixa escolaridade tornaria inviável a aquisição por financiamento próprio.

Nesse sentido, a condição sorológica da criança soropositiva demanda ao familiar cuidados específicos, principalmente, na manutenção da adesão à TARV. Sendo assim, o apoio para pontecialização dos atributos da família no cuidado cotidiano, proporcionado pelos profissionais da saúde e da educação, é imprescindível. Isso contribui para que o familiar compreenda a necessidade e a importância do cuidado, de forma que possa desempenhá-lo de maneira segura, minimizando o estresse que a rotina acarreta.

A falta de autonomia das crianças demanda ao familiar uma atenção especial, auxiliando-as na adesão à TARV. Torna-se necessário que a adesão seja obtida por meio do conhecimento dos benefícios que acarretará para o seu bem-estar. Nesse sentido, aponta-se a necessidade de profissionais da saúde desenvolvem ações de educação em saúde, propondo uma transição gradativa para que a criança desenvolva autonomia de modo não impositivo.

O cuidar da criança com HIV/aids condiciona o familiar a uma rotina desgastante pelo acúmulo de inúmeras responsabilidades que, somadas a fatores socioeconômicos e à falta de conhecimentos específicos da doença, comprometem a adesão à TARV. Essas dificuldades podem ser minimizadas pela atuação 
da equipe de saúde multiprofissional, por meio de orientação coerente com o cotidiano e com o nível de compreensão familiar. E, compartilhando informações acerca da doença, ajudando a família a identificar os medicamentos e comprometer-se com o tratamento.

Dessa forma, o papel da enfermagem ganha importância nas consultas individuais e coletivas, as quais propiciam abordar, por meio da escuta e do aconselhamento, essas crianças e familiares, numa perspectiva educativa a fim de fortalecer a compreensão e adesão à TARV.

\section{REFERÊNCIAS}

1. Costa JSD, Victora CG. O que é "um problema de saúde pública"? Rev. bras. epidemiol. [Internet] 2006;9(1) [acesso em 30 ago 2012]. Disponível: http://dx.doi. org/10.1590/S1415-790X2006000100018

2. Silva SFR, Pereira MRP, Neto RM, Ponte MF, Ribeiro IF, Costa PFTF et al. Aids no Brasil: uma epidemia em transformação. Rev. Bras. Anal. Clín. 2010;42(3):209-12.

3. Ministério da Saúde (BR). Secretaria de Vigilância em Saúde. Programa Nacional de DST e AIDS. Boletim Epidemiológico AIDS/DST 2012. [acesso em 02 maio 2012]. Disponível: http://www.aids.gov.br/sites/ default/files/anexos/publicacao/2011/50652/boletim_ aids_2011_final_m_pdf_26659.pdf

4. Padoin SMM, Paula CC, Tronco CS, Ribeiro AC, Santos EEP, Hoffmann IC et al. Crianças que têm HIV/ AIDS e seus familiares/ cuidadores: experiência de acompanhamento interdisciplinar. Rev. Saúde (Santa Maria). 2009;35(2):51-6.

5. Schaurich D, Medeiros HMF, Motta MGC. A cronicidade no processo saúde-doença: repensando a epidemia da AIDS após os anti-retrovirais. Rev. enferm. UERJ. 2006; 14(3):455-62.

6. Schaurich D, Medeiros HMF, Motta MGC. Vulnerabilidades no viver de crianças com AIDS. Rev. enferm. UERJ. 2007;15(2):284-90.

7. Guerra CPP, Seidl EMF. Crianças e adolescentes com HIV/Aids: revisão de estudos sobre revelação do diagnóstico, adesão e estigma. Paidéia. 2009;19(42):59-65.

8. Mendes KD, Silveira RC, Galvão CM. Revisão integrativa: método de pesquisa para a incorporação de evidências na saúde e na enfermagem. Texto Contexto Enferm. 2008;17(4): 758-64.
9. Ursi ES, Galvão CM. Prevenção de lesões de pele no perioperatório: revisão integrativa da literatura. Rev. Latino-Am. Enfermagem. 2006;14(1):124-31.

10. Pompeo DA, Rossi LA, Galvão CM. Revisão integrativa: etapa inicial do processo de validação de diagnóstico de enfermagem. Acta Paul. Enferm. 2009;22(4):434-8.

11. Badgilign S, Deribew A, Amberbir A, Deribe K. Adherence to highly active antiretroviral therapy and its correlates among HIV infected pediatric patients in Ethiopia. BMC Pediatrics [Internet] 2008;8:53 [acesso em 10 ago 2012]. Disponível: http://www.biomedcentral. com/1471-2431/8/53

12. Botene DZA, Pedro ENR. Implicações do uso da terapia antiretroviral no modo de viver de crianças com Aids. Rev Esc Enferm USP. 2011;45(1):108-15.

13. Gomes AMT, Cabral IE. Entre dose e volume: o princípio da matemática no cuidado medicamentoso à criança HIV positiva. Rev. enferm. UERJ. 2009;17(3):332-7.

14. Feitosa AC, Lima HJA, Caetano JA, Andrade LM, Beserra EP. Terapia anti-retroviral: fatores que interferem na adesão de crianças com HIV/AIDS. Esc. Anna Nery. 2008;12(3):515-21.

15. Trombini ES, Schermann LB. Prevalência e fatores associados à adesäo de crianças na terapia antiretroviral em três centros urbanos do sul do Brasil. Cienc. saúde colet. 2010;15(2):419-25.

16. White YR, Pierre RB, Steel-Duncan J, Palmer P, EvansGilbert T, Moore J et al. Adherence to antiretroviral drug therapy in children with HIV/AIDS in Jamaica. West Indian Med. J. 2008;57(3):231-7.

17. Bracher L, Valerius NH, Rosenfeldt V, Herlin T, Fisker $\mathrm{N}$, Nielsen $\mathrm{H}$ et al. Long-term effectiveness of highly active antiretroviral therapy (HAART) in perinatally HIV-infected children in Denmark. Scandinavian Journal of Infectious Diseases. 2007;39:799-804.

18. Ferreira DC, Souza LR, Berbert SHP, Reis HLB, Godefroy P, Volga G et al. Manifestações clínicas em crianças infectadas pelo HIV na era HAART: um estudo seccional. R. Ci. méd. biol. 2009;8(1):5-13.

19. Frota MA, Ramos RP, Mourão SJG, Vasconcelos VM, Martins MC, Araújo MAL. Cuidado à criança com HIV: percepção do cuidador. Acta. Sci. Health Sci. 2012;34(1):39-45.

20. Machado FC, Souza IPR, Tura LFR, Castro GF. Adesão 
a um programa de atenção à saúde bucal para crianças e adolescentes infectados pelo HIV e atitude dos responsáveis. Ciênc. saúde colet. 2008;13(6):1851-7.

21. Sousa AM, Lyra A, Araújo CCF, Pontes JL, Freire RC, Pontes TL. A política de AIDS no Brasil: uma revisão de literatura. JMPHC. 2012;3(1):62-6.

22. Neves ET, Cabral IE. Empoderamento da mulher cuidadora de crianças com necessidades especiais de saúde. Texto Contexto Enferm. 2008;17(3):552-60.

23. Salles CMB, Ferreira EAP, Seidl EMF. Adesão ao tratamento por cuidadores de crianças e adolescentes soropositivos para o HIV. Psic.: Teor. e Pesq. 2011;27(4):499-506.

24. Silva ALCN, Waidman MAP, Marcon SS. Adesão e não adesão a terapia antirretroviral: as duas faces de uma mesma vivência. Rev Bras Enferm. 2009;62(2):213-20.

25. Gomes AMT, Cabral IE. O cuidado medicamentoso à criança com HIV: desafios e dilemas de familiares cuidadores. Rev Bras Enferm. 2009;62(2):252-7.

26. Seidl EMF, Rossi WS, Viana KF, Meneses AKF, Meireles E. Crianças e adolescentes vivendo com HIV/Aids e suas famílias: aspectos psicossociais e enfrentamento. Psic.: Teor. e Pesq. 2005;21(3):279-88.

27. Freitas HMB, Backes DS, Pereira ADA, Ferreira CLL, Marchiori MRC, Souza MHT et al. Significados que os profissionais de enfermagem atribuem ao cuidado de crianças institucionalizadas com aids. Texto Contexto Enferm. 2010;19(3):511-7.

28. Calais LB, Jesus MAGS. Desvendando olhares: infância e AIDS nos discursos da sociedade. Psicol. soc. 2011;23(1):85-93.

29. Martin SM, Elliott-DeSorbo DK, Wolters PL, ToledoTamula MA, Roby G, Zeichner S et al. Patient, caregiver and regimen characteristics associated with adherence to highly active antiretroviral therapy among HIV-infected children and adolescents. Pediatr Infect Dis J. [Internet] 2007;26(1) [acesso em 19 ago 2012]. Disponível: http://www.deepdyve.com/lp/ medline-abstracts/patient-caregiver-and-regimencharacteristics-associated-with-4bViK764

30. Pieri FM, Laurenti R. HIV/AIDS: perfil epidemiológico de adultos internados em hospital universitário. Cienc. cuid. saude. 2012;11(supl.):144-52.

31. Ribeiro AC, Paula CC, Neves ET, Padoin SMM. Perfil clínico de adolescentes que têm AIDS. Cogitare enferm. 2010;12(2):256-62.

32. Polejack L, Seidl EMF. Monitoramento e avaliação da adesão ao tratamento antirretroviral para HIV/ aids: desafios e possibilidades. Ciênc. saúde colet. 2010;15(Supl.1): 1201-8.

33. Gomes AMT, Cabral IE. Ocultamento e silenciamento familiares no cuidado à criança em tratamento antiretroviral. Rev Bras Enferm. 2010;63(5):719-26.

34. Paula CC, Cabral IE, Souza ÍEO. O (não)dito da AIDS no cotidiano de transição da infância para a adolescência Rev Bras Enferm. 2011;64(4):658-64.

35. Paula CC, Cabral IE, Souza ÍEO. O cotidiano do ser-adolescendo com aids: movimento ou momento existencial? Esc. Anna Nery. 2009;13(2):632-9.

Cogitare Enferm. 2013 Abr/Jun; 18(2):379-86 